\title{
Reproductive and hormonal risk factors for postmenopausal luminal, HER2-overexpressing, and triple-negative breast cancer
}

\author{
Amanda I. Phipps, MPH ${ }^{1,3}$, Kathleen E. Malone, PhD ${ }^{1,3}$, Peggy L. Porter, MD ${ }^{2,4}$, Janet R. \\ Daling, $\mathbf{P h D}^{1,3}$, and Christopher I. Li, MD, $\mathbf{P h D}^{1,3}$ \\ ${ }^{1}$ Division of Public Health Sciences, Fred Hutchinson Cancer Research Center, Seattle, WA \\ ${ }^{2}$ Division of Human Biology, Fred Hutchinson Cancer Research Center, Seattle, WA \\ ${ }^{3}$ Department of Epidemiology, School of Public Health and Community Medicine, University of \\ Washington, Seattle, WA \\ ${ }^{4}$ Department of Pathology, School of Medicine, University of Washington, Seattle, WA
}

\begin{abstract}
Background-Molecular profiling studies have identified subtypes of breast cancer that can be approximately classified by estrogen receptor (ER), progesterone receptor (PR), and HER2-neu (HER2) expression. These molecular subtypes are prognostically significant, but differences in their etiologic profiles have not been established. Reproductive factors may plausibly be differentially related to risk of different breast cancer subtypes since these factors are presumed to impact exposure to endogenous sex hormones.
\end{abstract}

Methods-We pooled two population-based case-control studies of breast cancer in women aged 55-79 years, for an analysis including 1,476 controls and 1,023 luminal, 39 HER2-overexpressing, and 78 triple-negative cases. Polytomous logistic regression was used to compare each case group to controls.

Results-Associations varied by molecular subtype. Early age at menarche was only associated with risk of HER2-overexpressing disease [odds ratio $(\mathrm{OR})=2.7,95 \%$ confidence interval $(\mathrm{CI})$ : 1.4-5.5], while breastfeeding for 6 months or longer was only protective for luminal and triplenegative disease ( $\mathrm{OR}=0.8,95 \%$ CI:0.6-1.0 and $\mathrm{OR}=0.5,95 \%$ CI: $0.3-0.9$, respectively). Both late age at menopause and use of estrogen plus progestin hormone therapy were only associated with risk of luminal disease ( $\mathrm{OR}=1.6,95 \% \mathrm{CI}$ : $1.1-2.2$, and $\mathrm{OR}=1.7,95 \% \mathrm{CI}: 1.3-2.1$, respectively). No differences in risks associated with parity or age at first live birth were observed by subtype.

Conclusions-Certain reproductive factors may have a greater impact on risk of certain molecular subtypes of disease compared to others. Future studies that further define the etiology of breast cancer subtypes will add to our biological understanding of the disease.

\section{Keywords}

breast cancer; postmenopausal; triple-negative; luminal; HER2; breastfeeding; parity; menarche

\section{INTRODUCTION}

Gene expression studies have identified and validated the existence of five "intrinsic" breast cancer subtypes: luminal A, luminal B, HER2-overexpressing, basal-like, and unclassified.

Corresponding Author: Amanda I. Phipps, MPH Fred Hutchinson Cancer Research Center 1100 Fairview Ave. N. P.O. Box 19024 Seattle, WA 98109-1024 Tel: (206) 667-2878 Fax: (206) 667-5948 aphipps@ fhcrc.org . 
$1^{-3} 3$ Luminal A and B tumors are characterized by expression of the estrogen receptor $\underline{\alpha}$ (ER), typically accompanied by expression of the progesterone receptor (PR). Non-luminal subtypes are characterized by a lack of hormone-receptor expression and can be distinguished by the expression of protein tyrosine kinase HER2-neu (HER2): HER2 is upregulated in HER2-overexpressing tumors while basal-like and unclassified tumors both have the so-called 'triple-negative' phenotype (i.e., ER-/PR-/HER2-).4' 5 Clinical differences between these breast cancer subtypes have been well-described,2, 4, 6 Compared to luminal tumors, both HER2-overexpressing and triple-negative tumors have a poorer prognosis. However, differences in the epidemiology of these subtypes are not well-defined. Existing literature suggest that basal-like tumors are more common among AfricanAmerican women, 4,7 premenopausal women, 4,8 and women with BRCA1 mutations.9, 10 Further assessment of risk factors stratified by tumor subtype is important to identify disease-specific associations that might be masked by grouping together luminal, HER2overexpressing, and triple-negative breast cancers.

The focus of this study is the relationship between reproductive characteristics and risks of luminal, HER2-overexpressing, and triple-negative breast cancer. Age at menarche and menopause, parity, age at first live birth, and breastfeeding are all established breast cancer risk factors which influence risk primarily through hormonal mechanisms. 11 It has been suggested that factors such as these, that are related to endogenous hormone exposure, are more strongly associated with risk of ER+ (luminal) disease, while risk factors for ER- (nonluminal) disease are more likely to involve non-hormonal mechanisms.12-14 A metaanalysis of epidemiologic studies of breast cancer with ER and PR tumor marker data found that parity and age at first birth were associated with risk of hormone receptor-positive but not hormone receptor-negative disease. 15 However, data on differences in the associations between these factors and risk of different molecularly defined breast cancer subtypes are quite limited. Here we examine differences in the associations between reproductive and hormonal characteristics and risk of breast cancer by disease subtype, defined by joint ER/ PR/HER2 status.

\section{METHODS}

Given the rarity of HER2-overexpressing and triple-negative tumors, cases and controls were pooled from two population-based case-control studies of breast cancer. Both studies were approved by the Fred Hutchinson Cancer Research Center (FHCRC) institutional review board, and written informed consent was obtained from all study participants. Both of these studies have been described in detail elsewhere.16, 17 Briefly, cases for both studies were identified through the Cancer Surveillance System (CSS) of western Washington State as women with incident invasive breast cancer and no prior history of in situ or invasive breast cancer, residing in King, Pierce, or Snohomish counties at the time of diagnosis. For the earlier of these studies, 16 eligible cases were diagnosed between April 1, 1997 and May 31, 1999, were age 65-79 at diagnosis, and had a Health Care Financing Administration (HCFA) record. Potential controls without a prior breast cancer were identified through HCFA records and frequency matched to cases on age. The study base for the more recently completed study was identical to that of the earlier study with respect to geography and case ascertainment protocols, although cases eligible for the more recently completed study were diagnosed with breast cancer between January 1, 2000 and March 31, 2004, at age 55-74 years. Additionally, given that the original purpose of the latter study was to identify differences in risk factor associations by tumor histology, cases were sampled on the basis of histology; specifically, all lobular cases and a random $25 \%$ sample of ductal cases were eligible. 17 Controls for this study were identified through random-digit dialing 18 and frequency matched to cases on age. Response rates were similar for both studies $(80.6 \%$ and $73.6 \%$ for cases and controls, respectively, in the earlier study, and $83.5 \%$ and $71.1 \%$ for 
cases and controls, respectively, in the more recent study), and a total of 2,019 eligible cases and 1,476 eligible controls were enrolled and interviewed. The present analysis was restricted to controls and ductal cases $(n=1,233)$, given the rarity of ER negativity in lobular disease $(<10 \%)$ and the oversampling of lobular cases in the more recent study.

The pooled studies used similar protocols and materials. Study questionnaires were administered in-person and obtained data on a number of risk factors including lifestyle factors, reproductive history, hormone use, and family history of cancer. Women were asked about exposures occurring before their reference date, defined as the date of diagnosis for cases and assigned to controls based on the expected distribution of case reference dates.

Data on ER, PR, and HER2 expression status were identified from CSS records. Additionally, centralized pathology testing at the FHCRC had been completed for a sample of cases as part of the protocol from the more recent study; thus, for a subset of cases for whom data on HER2 expression were not available from CSS records, this information was derived from results of centralized testing $(\mathrm{n}=97)$. All ER and PR results, and a majority of HER2 results, were based on immunohistochemistry (IHC) testing. When available, HER2 data from FISH assays recorded in pathology reports $(n=32)$ were given precedence over IHC results. Overall, ER and PR data were available for 1,179 cases $(96 \%), 883(75 \%)$ of whom were ER+ and PR+, 148 (13\%) were ER- and PR-, 139 (12\%) were ER+ and PR-, and $9(<1 \%)$ were ER- but PR+. Data for HER2 were available for 847 cases $(69 \%)$ in total, of whom $730(86 \%)$ were HER2- and 117 (14\%) were HER2+. Classification of case subtypes was made on the basis of ER, PR, and HER2 data as follows: luminal (ER+), HER2-overexpressing (ER-/PR-/HER2+), and triple-negative (ER-/PR-/HER2-). Cases with the rare ER-/PR+ phenotype were excluded $(n=9)$, as were cases with insufficient tumor marker data ( $\mathrm{n}=52$ cases missing ER data, $\mathrm{n}=32 \mathrm{ER}-/ \mathrm{PR}$ - cases missing HER2 data). Final analyses were based on 1,476 controls and 1,140 cases [1,023 (90\%) luminal, 39 (3\%) HER2-overexpressing, and 78 (7\%) triple-negative].

We used polytomous logistic regression 19 to compare luminal, HER2-overexpressing, and triple-negative case groups to a common control group; this approach is directly comparable to performing a series of simple binary logistic regression models for different case-control comparisons. All analyses were adjusted for age (continuous) and reference year (continuous); because there was no overlap in reference years between the two pooled studies, adjustment for reference year resulted in implicit adjustment for study population. The following non-reproductive breast cancer risk factors were evaluated as potential confounders: education level ( $\leq$ high school degree/some college/college graduate), smoking status (yes/no), alcohol consumption (non-drinker/ $\leq 7$ drinks per week/> 7 drinks per week), and family history of breast cancer in first-degree relatives (yes/no). We similarly evaluated confounding between each of the reproductive factors under study. Adjustment for potential non-reproductive confounders did not alter risk estimates by more than $10 \%$ for any tumor subtype. Adjustment for age at first live birth and breastfeeding history did, however, have an effect on estimates for number of live births. Similarly, adjustment for type of menopause impacted effect estimates for hormone therapy (HT) use and age at menopause. Therefore, final multivariate analyses for number of live births, age at first live birth, and breastfeeding were co-adjusted for one another and restricted to parous women with data for each of these variables ( $n=1,226$ controls, 802 luminal cases, 32 HER2overexpressing cases, 62 triple-negative cases). Final multivariate analyses for HT use and age at menopause were adjusted for type of menopause. Analysis of age at menopause was restricted to women who experienced menopause (natural or due to bilateral oophorectomy) at a known age ( $\mathrm{n}=1,087$ controls, 763 luminal cases, 27 HER2-overexpressing cases, 54 triple-negative cases). Tests for trend were also conducted, modeling variable categories with a single linear term. Heterogeneity of effect estimates between case groups was 
examined through a series of polytomous regression models comparing HER2overexpressing and triple-negative case groups to the luminal case group. All statistical analyses were performed using STATA/SE version 9 (StataCorp, College Station, Texas).

\section{RESULTS}

Compared to controls and the other case groups, luminal cases were somewhat more likely to have a first-degree family history of breast cancer and to be diagnosed at an early stage, HER2-overexpressing cases were less likely to be non-Hispanic whites and to be college graduates, and triple-negative cases were more likely to be younger (Table 1). With respect to reproductive characteristics, relationships with menarche and parity-related events varied somewhat by tumor subtype (Table 2). We found that early age at menarche was only associated with risk of HER2-overexpressing disease (OR age $<13$ vs. $\geq 13=2.7,95 \%$ CI: 1.4-5.5). Compared to nulliparous women, women with one or more live births experienced a modestly lower risk of luminal disease that was within the limits of chance $(\mathrm{OR}=0.8,95 \%$ CI: 0.6-1.0). Among parous women, neither number of live births nor age at first live birth were strongly related to risk of any tumor subtype. Women who breastfed for $\geq 6$ months had reduced risks of both luminal $(\mathrm{OR}=0.8,95 \% \mathrm{CI}$ : 0.7-1.0) and triple-negative disease $(\mathrm{OR}=0.5,95 \%$ CI: 0.3-0.9) relative to parous women who had never breastfed. Risk of luminal breast cancer was lower among women who had a surgical menopause compared to women who experienced a natural menopause (OR=0.7, 95\% CI: 0.5-0.9), and higher among women who had a late age at menopause (OR for age $\geq 55$ vs. $<45=1.6,95 \% \mathrm{CI}$ : 1.1-2.2) or were current users of estrogen and progestin hormone therapy ( $\mathrm{OR}=1.7,95 \% \mathrm{CI}$ : 1.3-2.1). No statistically significant associations between menopausal status, age at menopause, or hormone therapy use and risks of either HER2-overexpressing or triplenegative tumors were observed. However, there was a suggestion that age at menopause was positively associated with risk of HER2-overexpressing disease ( $\mathrm{p}$ for linear trend $=0.07$ ).

\section{DISCUSSION}

It is important to acknowledge the limitations of this study before interpreting its results. Despite combining two studies, the statistical power of our analyses is limited by the inclusion of only 78 triple-negative and 39 HER2-overexpressing cases. We also had to exclude 84 (7\%) potentially eligible cases who lacked sufficient tumor marker data to be classified into a subtype. In addition, we were unable to distinguish between basal-like and unclassified cases, both of which have the triple-negative phenotype, because we lacked data on EGFR and cytokeratin 5/6 expression, which are necessary to distinguish between these two subtypes. It is also possible that some misclassification of case subtypes resulted from the use of ER, PR, and HER2 data taken from multiple laboratories in the Seattle-Puget Sound region, as the assays and practices for reading and interpreting results vary across institutions and pathologists; however, it is reasonable to assume any such misclassification is non-differential with respect to reproductive factors.

Consistent with a number of prior studies that have investigated the association between reproductive factors and risk of hormone-receptor positive breast cancer, $13^{-} 15^{,}, 20^{-} 22$ we observed that current users of combined estrogen plus progestin preparations of HT and women who had a later age at menopause had an increased risk of luminal breast cancer, and women who breastfed had a decreased risk of luminal breast cancer. We also found that certain reproductive and hormonal characteristics were related to risk of HER2overexpressing and triple-negative tumors. Age at menarche was only associated with risk of HER2-overexpressing disease, and women who breastfed had a reduced risk of triplenegative disease. Only two prior studies have assessed differences in the associations between reproductive factors and breast cancer risk according to molecularly-defined breast 
cancer subtypes: the Polish Breast Cancer Study (PBCS)8, 23 and the Carolina Breast Cancer Study (CBCS).23 There are important differences between these studies and ours as both the PBCS and the CBCS included premenopausal and postmenopausal women, and the CBCS also included a substantial proportion of African Americans (41\%). Thus, it is difficult to compare our findings to these studies due to differences in age and race/ethnicity distributions, and because both prior studies, as well as ours, are relatively underpowered by the inclusion of limited numbers of basal-like ( $n=95$ and $n=225$ ) and HER2-overexpressing cases $(n=61$ and $n=116)$. Our results are consistent with the CBCS in their observation that breastfeeding for $\geq 4$ months was associated with a reduced risk of basal-like breast cancer (OR=0.7, 95\% CI: 0.4-0.9). Contrary to our findings however, both the CBCS and the PBCS observed an elevated risk of basal-like breast cancer among nulliparous relative to parous women.

While definitive conclusions regarding which risk factors are more strongly related to certain molecularly-defined subtypes of breast cancer cannot be made from this study, our data do support the premise that risk factor profiles vary by breast cancer subtype and that hormonal risk factors have a greater impact on luminal type breast cancers than HER2overexpressing or triple-negative tumors. The observation that breastfeeding has now been shown to reduce risk of triple-negative disease in two studies is intriguing, but the biological basis for this association is unclear and this finding requires further replication. Given the well-defined clinical differences between breast tumor subtypes, the relatively poor prognoses of HER2-overexpressing and triple-negative disease, 2, 4, 6 and the paucity of existing epidemiologic data, further research is needed to clarify the epidemiology of these tumors because at present no consistent risk factors for either of these two subtypes have been identified.

\section{Acknowledgments}

The studies in this analysis were both supported by the National Cancer Institute (NCI) through contracts with the Fred Hutchinson Cancer Research Center (R01 CA072787 and R01 CA85913). The work of A.I.P. in this publication was made possible by the National Center for Research Resources (NCRR, Grant Number 1 TL1 RR02501601), a component of the National Institutes of Health (NIH) and NIH Roadmap for Medical Research.

\section{REFERENCES}

1. Perou C, Sorlie T, Eisen M, et al. Molecular portraits of human breast tumours. Nature. 2000; 406:747-52. [PubMed: 10963602]

2. Sorlie T, Perou C, Tibshirani R, et al. Gene expression patterns of breast carcinomas distinguish tumor subclasses with clinical implications. Proc Natl Acad Sci U S A. 2001; 98:10869-74. [PubMed: 11553815]

3. Sorlie T, Tibshirani R, Parker J, et al. Repeated observation of breast tumor subtypes in independent gene expression data sets. Proc Natl Acad Sci U S A. 2003; 100:8418-23. [PubMed: 12829800]

4. Carey L, Perou C, Livasy C, et al. Race, breast cancer subtypes, and survival in the Carolina Breast Cancer Study. Jama. 2006; 295:2492-502. [PubMed: 16757721]

5. Nielsen T, Hsu F, Jensen K, et al. Immunohistochemical and clinical characterization of the basallike subtype of invasive breast carcinoma. Clin Cancer Res. 2004; 10:5367-74. [PubMed: 15328174]

6. Carey L, Dees E, Sawyer L, et al. The triple negative paradox: primary tumor chemosensitivity of breast cancer subtypes. Clin Cancer Res. 2007; 13:2329-34. [PubMed: 17438091]

7. Bauer K, Brown M, Cress R, Parise C, Caggiano V. Descriptive analysis of estrogen receptor (ER)negative, progesterone receptor (PR)-negative, and HER2-negative invasive breast cancer, the socalled triple-negative phenotype: a population-based study from the California cancer Registry. Cancer. 2007; 109:1721-8. [PubMed: 17387718] 
8. Yang X, Sherman M, Rimm D, et al. Differences in risk factors for breast cancer molecular subtypes in a population-based study. Cancer Epidemiol Biomarkers Prev. 2007; 16:439-43. [PubMed: 17372238]

9. Lakhani S, Van De Vijver M, Jacquemier J, et al. The pathology of familial breast cancer: predictive value of immunohistochemical markers estrogen receptor, progesterone receptor, HER-2, and p53 in patients with mutations in BRCA1 and BRCA2. J Clin Oncol. 2002; 20:2310-8. [PubMed: 11981002]

10. Foulkes W, Stefansson I, Chappuis P, et al. Germline BRCA1 mutations and a basal epithelial phenotype in breast cancer. J Natl Cancer Inst. 2003; 95:1482-5. [PubMed: 14519755]

11. Kelsey J, Gammon MD, John E. Reproductive factors and breast cancer. Epidemiol Rev. 1993; 15:36-47. [PubMed: 8405211]

12. Huang W, Newman B, Millikan R, Schell M, Hulka B, Moorman P. Hormone-related factors and risk of breast cancer in relation to estrogen receptor and progesterone receptor status. Am $\mathrm{J}$ Epidemiol. 2000; 151:703-14. [PubMed: 10752798]

13. Potter J, Cerhan J, Sellers T, et al. Progesterone and estrogen receptors and mammary neoplasia in the Iowa Women's Health Study: How many kinds of breast cancer are there? Cancer Epidemiol Biomarkers Prev. 1995; 4:319-26. [PubMed: 7655325]

14. Rosenberg L, Einarsdottir K, Friman E, et al. Risk factors for hormone receptor-defined breast cancer in postmenopausal women. Cancer Epidemiol Biomarkers Prev. 2006; 15:2482-8. [PubMed: 17164374]

15. Ma H, Bernstein L, Pike MC, Ursin G. Reproductive factors and breast cancer risk according to joint estrogen and progesterone receptor status: a meta-analysis of epidemiological studies. Breast Cancer Res. 2006; 8:R43. [PubMed: 16859501]

16. Li C, Malone K, Porter P, et al. Relationship between long durations and different regimens of hormone therapy and risk of breast cancer. JAMA. 2003; 289:3254-63. [PubMed: 12824206]

17. Li C, Malone K, Porter P, et al. Relationship between menopausal hormone therapy and risk of ductal, lobular, and ductal-lobular breast carcinomas. Submitted to Cancer Epidemiol Biomarker Prev.

18. Waksberg J. Sampling methods for random digit dialing. J Am Stat Assoc. 1978; 73:40-6.

19. Begg C, Gray R. Calculation of polychotomous logistic regression parameters using individualized regressions. Biometrika. 1984; 71

20. Ursin G, Bernstein L, Lord SJ, et al. Reproductive factors and subtypes of breast cancer defined by hormone receptor and histology. Br J Cancer. 2005; 93:364-71. [PubMed: 16079783]

21. Nichols HB, Trentham-Dietz A, Love RR, et al. Differences in breast cancer risk factors by tumor marker subtypes among premenopausal Vietnamese and Chinese women. Cancer Epidemiol Biomarkers Prev. 2005; 14:41-7. [PubMed: 15668474]

22. Tjonneland A, Christensen J, Thomsen BL, et al. Hormone replacement therapy in relation to breast carcinoma incidence rate ratios: a prospective Danish cohort study. Cancer. 2004; 100:2328-37. [PubMed: 15160335]

23. Millikan R, Newman B, Tse C, et al. Epidemiology of basal-like breast cancer. Breast Cancer Res Treat. 2007 
TABLE 1

Distribution of demographic and risk factors by case status and tumor type

\begin{tabular}{|c|c|c|c|c|}
\hline & $\begin{array}{c}\text { Controls } \\
(\mathbf{N}=1,476) \\
\mathbf{N}(\%)\end{array}$ & $\begin{array}{c}\text { Luminal cases } \\
(\mathbf{N}=\mathbf{1 , 0 2 3}) \\
\mathbf{N}(\%)\end{array}$ & $\begin{array}{c}\text { Her2- } \\
\text { overexpressing } \\
\text { cases } \\
(\mathbf{N}=\mathbf{3 9}) \\
\mathbf{N}(\%)\end{array}$ & $\begin{array}{c}\text { Triple-negative } \\
\text { cases } \\
(\mathbf{N}=78) \\
\mathbf{N}(\%)\end{array}$ \\
\hline \multicolumn{5}{|l|}{ Age } \\
\hline $55-64$ & $258(17)$ & $226(22)$ & $12(31)$ & $28(36)$ \\
\hline $65-74$ & $922(62)$ & $621(61)$ & $19(49)$ & $40(51)$ \\
\hline$\geq 75$ & $296(20)$ & $176(17)$ & $8(21)$ & $10(13)$ \\
\hline MEAN & 69.3 & 68.6 & 67.6 & 66.0 \\
\hline \multicolumn{5}{|l|}{ Race/Ethnicity } \\
\hline Non-Hispanic White & $1348(91)$ & $956(93)$ & $32(82)$ & $72(92)$ \\
\hline African American & $45(3)$ & $17(2)$ & $2(5)$ & $3(4)$ \\
\hline Asian/Pacific Islander & $38(3)$ & $29(3)$ & $3(8)$ & $1(1)$ \\
\hline Other & $45(3)$ & $21(2)$ & $2(5)$ & $2(3)$ \\
\hline \multicolumn{5}{|l|}{ Education, yrs } \\
\hline$\leq 12$ & $698(47)$ & $450(44)$ & $18(46)$ & $34(44)$ \\
\hline $13-15$ & $468(32)$ & $336(33)$ & $17(44)$ & $28(36)$ \\
\hline$\geq 16$ & $309(21)$ & $237(23)$ & $4(10)$ & $16(21)$ \\
\hline \multicolumn{5}{|c|}{$\begin{array}{l}\text { First-degree family history } \\
\text { of breast cancer }\end{array}$} \\
\hline No & $1151(83)$ & $734(76)$ & $33(85)$ & $62(87)$ \\
\hline Yes & $230(17)$ & $226(24)$ & $6(15)$ & $9(13)$ \\
\hline Missing & 95 & 63 & 0 & 7 \\
\hline \multicolumn{5}{|l|}{$\underline{\text { Stage at diagnosis }}$} \\
\hline$\underline{I}$ & $\underline{\mathrm{N} / \mathrm{A}}$ & $\underline{715(70)}$ & $\underline{22(56)}$ & $\underline{45(59)}$ \\
\hline II & & $\underline{271(27)}$ & $\underline{12(31)}$ & $\underline{25(33)}$ \\
\hline$\underline{\text { III }}$ & & $\underline{29(3)}$ & $\underline{5(13)}$ & $\underline{6(8)}$ \\
\hline
\end{tabular}


Phipps et al.

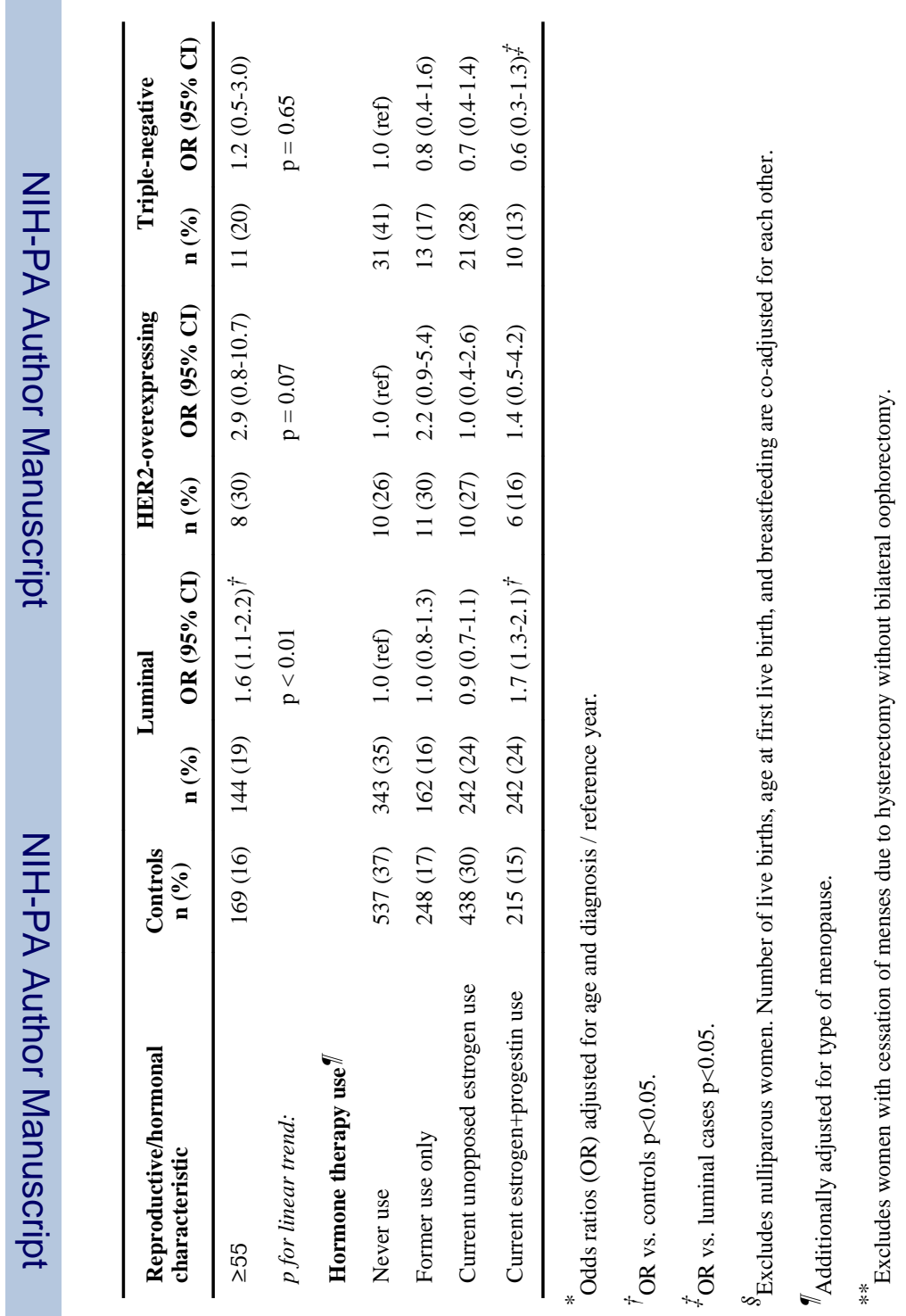

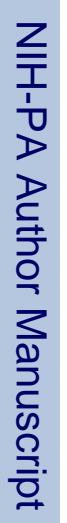

